\title{
Study on the Adsorption Performance of Various Adsorbents on Hazardous and Noxious Substances
}

\author{
Hongyan Wang, Wen Chang and Yujun Tian* \\ China Waterborne Transport Research Institute, Beijing, China
}

\begin{abstract}
Hazardous and noxious substances (HNS) leakage from ship accidents has been one of the major sources of ship pollutions. Due to the varieties of HNS, the complexity of physical and chemical properties, the harmfulness of potential accidents, and the difficulty of accident prevention, HNS leakage could cause huge social and environmental impacts. HNS emergency response methods and technologies are currently in the early and exploring stage. As a big HNS import and export country with the increasing transportation volume, the risk of HNS pollution accidents in the ports has been significantly increasing over the recent years. However, few studies have been conducted regarding the HNS response technology, especially to the adsorption performance of various adsorbents against HNS. Therefore, this study aims to calculate the degree of adsorption of different adsorbents by selecting the representative HNS, which are commonly transported in the volume, for example, benzene, styrene, and vegetable oil. For inert substances with low saturated vapor pressure (vegetable oil and styrene), the degree and rate of adsorption of the investigated materials were found to be inversely proportional to the mass per unit area (area density). Moreover, these investigated adsorbents were shown to exhibit a good adsorption retention rate against inert substances. This study can provide the technical standard to select the most suitable adsorbents for the relevant HNS leakage.
\end{abstract}

\section{Introduction}

\subsection{Background}

The Protocol on Preparedness, Response and Co-operation to pollution incidents by Hazardous and Noxious Substances(OPRC-HNS Protocol) follows the principles of the International Convention on Oil Pollution Preparedness, Response and Cooperation(OPRC), 1990 [1] and was formally adopted by States already Party to the OPRC Convention at a Diplomatic Conference held at International Maritime Organization(IMO) headquarters in London in March 2000. The OPRC-HNS Protocol, designed for preparedness and response,

\footnotetext{
* Corresponding author: wanghy@wti.ac.cn
} 
defines HNS as a substance other than oil which if introduced into the marine environment is likely to create hazards to human health, to harm living resources and marine life, to damage amenities or to interfere with other legitimate uses of the sea [2]. Like the OPRC Convention, the HNS Protocol aims to provide a global framework for international cooperation in combating major incidents or threats of marine pollution [3].

\subsection{Characteristics of HNS leakage accident}

Due to the diversity of HNS varieties and different physical properties, HNSs exist in one or more of the following behaviors after the leakage into the water: dissolution, evaporation, floating, and sedimentation. The European Chemical Behavior Classification Standard (SEBC) divides HNS leakage into 5 major categories (gas, evaporator, floater, dissolver, sinker), and they are grouped into 12 groups according to ITOPF Technical Information [4]. HNS leakage involves potentially polluting objects including air, seawater surfaces, water bodies, and sea-floor. Also, the leakage indirectly affects the organisms and users in these ecosystems, leading to more serious damage to human health.

At present, there are more than 30,000 chemicals registered in the International Maritime Organization and there is an increasing trend of certain types. Most HNSs with strong biological toxicity tend to accumulate in organisms, leading to the great damage of the environment. Some HNSs will evaporate much faster than crude oil spills. The evaporation event could occur for several days, which means that the surrounding ecosystems are exposed to potentially dangerous chemical vapors [5]. Based on the above characteristics, the recovery of HNSs is challenging and complex. HNS leakage treatment emergency requires trained specialists who will handle fast recovery works. The disposal methods of HNS leakage accidents include the adsorption, recovery, chemical, combustion, and microbiological methods.

Table 1. HNS behavior in Water (ITOPF).

\begin{tabular}{ccc}
\hline Environmental Behavior & Category & Characteristics \\
\multirow{2}{*}{ Gas } & $\mathrm{G}$ & Gas \\
& $\mathrm{GD}$ & Gas, Dissolver \\
\hline \multirow{2}{*}{ Evaporator } & $\mathrm{E}$ & Evaporator \\
& $\mathrm{ED}$ & Evaporator, Dissolver \\
\hline \multirow{2}{*}{ Floater } & FE & Floater, Evaporator \\
& FED & Floater, Evaporator, Dissolver \\
& $\mathrm{F}$ & Floater \\
& FD & Floater, Dissolver \\
\hline \multirow{2}{*}{ Dissolver } & $\mathrm{DE}$ & Dissolver, Evaporator \\
& $\mathrm{D}$ & Dissolver \\
\hline \multirow{2}{*}{ Sinker } & $\mathrm{SD}$ & Sinker, Dissolver \\
& $\mathrm{S}$ & Sinker \\
\hline
\end{tabular}

Source: ITOPF

\section{Literature review}

The American Society for Testing and Materials (ASTM) has issued the Standard Test Methods for Sorbent Performance of Adsorbents F726-12 in 2012 [6]. This standard is used for laboratory determination (off-site determination) of the various materials used as 
absorbents to remove non-emulsified oils, other floating and non-melting liquids on the water surface. Performance indicators include water resistance, release rate, etc.

At the third working draft meeting of the International Organization for Standardization held in July 2015, the Korean Technology and Standards Agency proposed the "Ship and Marine Technology-Marine Environment Protection-Guidance on Design and Selection of Sorbents". The purpose of this standard is to assist manufacturers and customers in selecting the suitable adsorbent material according to the technical standards. The standard specifies the types of adsorbents with the specific selection criteria and characteristics [7].

The Canadian General Standards Board (CGSB), as a member of the Canadian National Standards System, is responsible for Canadian National Standards (NSC), Canadian General Standards Board (CGSB), Canadian Government Standards (GC), and International Standards (ISO). CGSB has been developing and providing services to private enterprises and the public sector with assistance in the specification, qualification, and certification of standards. CGSB has established the formulation for the national standards in more than 70 fields. The number of published standards has now reached nearly 350 . The formulation of the "Absorbent Material Used for Oils, Liquid Fuels and Water" [8] showed the adsorption performance of tested adsorbents on oils, liquid fuels, and water.

"Materials used for the control of liquid spillages- Part 2: Determination of water repellency or buoyancy for hydrophobic (oil sorbent) materials" [9] was commissioned by the British Technical Committee CII/63, which was established by the AEA Technology, British Oil Spill Control Association, British Industrial Safety Alliance, Chemical Industry Association, Environmental Protection Agency, Manufacturing Personal Safety Association, and other institutions. "Materials used for the control of liquid spillages-Part 1: Determination of sorbency" [10] specifies a test method for the adsorption performance of materials. This method ensures that the users can compare and select the suitable adsorbent materials under the specific application conditions to improve the speed of pollutant leakage emergency response.

The French Association for Standardization (AFNOR) is a French member of the French Standardization Organization, the International Standardization Organization, and the International Electro-technical Commission. AFNOR has formulated several technical standards for adsorbents, namely: "Determination of absorbency" [11], "Determination of the absorbency of all liquids" [12], and "Classification of absorbent products" [13].

Japan has been studying HNS leakage emergency technology since 2001 and has proposed the "2000 HNS Pollution Prevention, Response and Cooperation Protocol". The initial research included up to 4 years of laboratory testing of different adsorbents, where the Japan Maritime Disaster Prevention Center (MDPC) selected 35 types of HNS with a large volume for the test. In these tests, 14 compounds are water-soluble or heavy non-aqueous liquids, and comparable HNS volatile gases [14]. The reduction of different HNS volatile gas was compared to determine the extent of benzene, alkanes and esters absorption. This experimental method is still widely used as a test method for the adsorption performance of adsorbents.

"Sorbents for Ship" [15] was proposed and managed by the Maritime Safety Administration of the People's Republic of China. This standard specifies the physical and chemical properties of marine oil (such as adsorption and viscosity). "Oil Adsorption Boom" [16] is the standard for the transportation industry of the People's Republic of China, which was proposed and managed by the Maritime Safety Administration of the People's Republic of China. This standard specifies the method to test the selected materials which can adsorb floating oil on water. 
The Environmental Protection Center of the Ministry of Transport developed the experimental method for the determination of the adsorption performance of the MDHX400SS chemical adsorbent according to ASTM F726-12 "Standard Test Methods for Sorbent Performance of Adsorbents" [17].

"Study on the Application Performance of a New Type of Foam Material on the Adsorption of Oil and Hazardous Chemicals"[18] focuses on the foam adsorption performance against oils and other liquid hazardous chemicals under the different conditions. The adsorption performance was studied and analyzed comprehensively through the adsorption experiment and the release rate experiment on the aqueous solutions of water-soluble hazardous chemicals, and the adsorption of pure hazardous chemical liquid experiment, etc.

\section{Methods}

In this study, three specific experimental reagents were selected to simulate the actual HNS leakage in a laboratory environment. The adsorption performance of the selected adsorbents for HNS was quantified.

\subsection{Selection of experimental reagents}

The experimental reagents were selected according to the common HNS leakage from the ships in China. The selection is also based on the behavior of HNS after leaking into the water, the toxicity of HNS, and the existing standards and research.

HNSs were selected for the large transport volume and low toxicity according to the list of 20 major chemicals, which are most likely to cause high risks in HNS accidents determined by the IMO [19]. The shipping HNSs which are less harmful to the environment were used as the experimental reagent in this study. Benzene, styrene, and vegetable oil were selected according to these conditions, the properties of experimental reagents are shown in Table 2. In addition, the three substances not only can be transported in a large volume but also act as floating and volatile substances after leaking into the water surface. The saturated vapor pressures and the volatilization rates are significantly different from each other. The selected HNSs can be used for comparison of the performances of various adsorbents.

Table 2. Properties of experimental reagents used in the adsorption test.

\begin{tabular}{ccccc}
\hline $\begin{array}{c}\text { Experimental } \\
\text { reagents }\end{array}$ & Density $\left(\mathrm{mg} / \mathrm{cm}^{3}\right)$ & $\begin{array}{c}\text { Saturated vapor } \\
\text { pressure }(\mathrm{mmHg})\end{array}$ & Viscosity $(\mathrm{mPa} . \mathrm{s})$ & Behavior \\
\hline Vegetable & $915.0 \sim 937.5$ & 0.0465 & 8.5 & $\mathrm{~F}$ \\
Oil & 876.5 & 75 & 0.647 & $\mathrm{FE}$ \\
Benzene & 960.0 & 5.2 & 0.762 & $\mathrm{FE}$ \\
Styrene & & & & \\
\hline
\end{tabular}

\subsection{Selection of experimental adsorbents}

In this study, the selected adsorbents are made of polypropylene fibers, which exhibit good characteristics, such as high adsorption rate, low waste disposal cost, easy cleaning, wide chemical resistance, and no health hazard. The adsorbent materials are synthesized by the following steps: adding surfactants, dehydration, and de-oiling post-treatment. This process will prevent the decomposition after adsorbing HNSs. The adsorbents can be used to adsorb 
strong acid, strong alkaline, oily dirt, organic solvents, etc. Characteristics of experimental adsorbents are shown in Table 3.

Table 3. Characteristics of three selected adsorbents.

\begin{tabular}{cccc}
\hline Sample & Shapes & Raw material & $\begin{array}{c}\text { Mass area } \\
\text { ratio }\left(\mathrm{g} / \mathrm{m}^{2}\right)\end{array}$ \\
\hline $\mathrm{a}$ & slice & Polypropylene fiber & 400 \\
$\mathrm{~b}$ & slice & Polypropylene fiber & 170 \\
$\mathrm{c}$ & slice & Polypropylene fiber & 200 \\
\hline
\end{tabular}

\subsection{Selection of physical and chemical indicators}

Using the relevant standards, the characteristics of HNS leakage accidents and the role of adsorbents in an emergency were evaluated. Considering to achieve the highest treatment efficiency for overflowing HNS containment and HNS recovery, this study determines the degree of adsorption, the adsorption rate, and the adsorption retention rate as the physical and chemical indicators of adsorbents.

\subsubsection{The degree of adsorption}

The adsorption of the selected materials is mainly determined based on the amount of recovered HNSs. The adsorbent performance was estimated by measuring the mass of adsorbed HNS into the unit mass of adsorbents. The value is expressed in $\mathrm{ml} \mathrm{per} \mathrm{g}$.

\subsubsection{Adsorption rate}

The adsorption rate determination can provide the timeliness of the adsorbents to the HNS leakage emergency. The adsorption rate of the adsorbents is obtained by measuring the mass of adsorbed HNS over mass of the adsorbents in a specific time. The value is expressed in $\mathrm{g} / \mathrm{s} / \mathrm{g}$.

\subsubsection{Adsorption retention rate}

The adsorption retention rate can be used to evaluate the degree of retaining HNS adsorbed on the tested materials. This indicator can be used to provide whether the adsorbed HNS is re-eluted into the air or other media again. This parameter is also useful for establishing the guidelines for the transportation and disposal process. The adsorption retention rate is the ratio of adsorption amount of the adsorbents before and after the external force after the HNS adsorption. The value can be expressed in $\%$.

\subsection{Determination of experimental method}

During the evaluation, the adsorbents must make full contact with the HNS. Seven sets of parallel tests were performed for each type of adsorbents against each type of HNS. The initial mass $\left(m_{0}\right)$ of adsorbents and the mass $\left(m_{n}\right)$ after the adsorption is determined while the adsorbents are entirely in contact with HNS. Three sets of parallel tests were performed on each type of adsorbents for each type of HNS. 


\subsubsection{Determination of the degree of adsorption}

The test method is based on Article 5.5 of the British Standards Institution "Test Method for the Absorption Performance of Materials for Liquid Spill Control" (BS 7959-1:2004). The experimental material was weighed and placed into the experimental reagents. After adsorbing over the period $\left(t_{\mathrm{n}}\right)(20,40,60,80$ seconds), the adsorbents were removed and placed on the filter screen for drying, and to ensure no more liquid drips. The following formula expresses the degree of adsorption:

$$
S_{n}=\frac{m_{n}-m_{0}}{d_{\llcorner} m_{0}}
$$

Where:

$S_{\mathrm{n}}$ : the adsorption of the test materials, the value is expressed in $\mathrm{ml} / \mathrm{g}$;

$m_{\mathrm{n}}$ : the mass of the test adsorbents after adsorption, the value is expressed in $\mathrm{g}$;

$m_{0}$ : the initial mass of the test adsorbents, the value is expressed in $\mathrm{g}$;

$d_{L}:$ the density of the test reagent, the value is expressed in $\mathrm{g} / \mathrm{ml}$;

\subsubsection{Determination method of adsorption rate}

Weigh the experimental adsorbents and placed in the experimental reagents. The adsorption process will be carried out over a period of time () (20s, 40s, 60s, 80s). After the adsorption period, the adsorbents were removed and placed on the filter screen for drying. The dried adsorbents were weighed and noted as . The experiments were repeated three times. The following formula expresses the adsorption of the experimental materials:

$$
U_{n}=\frac{m_{n}-m_{0}}{t_{n} m_{0}}
$$

Where:

$U_{\mathrm{n}}$ : the adsorption rate of the tested adsorbents, the value is expressed in $\mathrm{g} / \mathrm{s} / \mathrm{g}$;

$t_{\mathrm{n}}:$ the adsorption time, the value is expressed in $\mathrm{s}$;

\subsubsection{Determination method of adsorption retention rate}

The test method refers to the Article 11.5.1 of the ASTM F726-12. The saturated test adsorbent mass $\left(m_{b}\right)$ was weighed and placed in a glass container filled with distilled water. Then the container was shaken for 5, 10, 20, 30, 50,60 and 90 min. Take the adsorbents out and place on the filter screen for drying. The test adsorbent mass $\left({ }^{m_{z}}\right)$ was weighed until no more liquid dripping. The adsorption retention rate of the adsorbents is calculated by $Q_{n}$, expressed in \%, calculated according to the formula (5): 


$$
Q_{n}=\frac{m_{z}}{m_{b}}
$$

\section{Results and discussions}

In this paper, the degree of adsorption, adsorption rate, and adsorption retention rate were fitted with the experimental time respectively. The fitting results are shown in Figure 1, Figure 2 and Figure 3. The optimal range of each parameter is shown in Table 4.

Table 4. The Adsorption Performance of Various Adsorbents against various HNS

\begin{tabular}{lccc}
\hline $\begin{array}{c}\text { Experimental } \\
\text { Reagents }\end{array}$ & $\begin{array}{c}\text { The degree of } \\
\text { adsorption }(\mathrm{ml} / \mathrm{g})\end{array}$ & $\begin{array}{c}\text { Adsorption Rate } \\
(\mathrm{g} / \mathrm{s} / \mathrm{g})\end{array}$ & $\begin{array}{c}\text { Adsorption retention } \\
\text { Rate }(\%)\end{array}$ \\
\hline Vegetable & $\geq 7.0$ & 0.3 & 95 \\
Oil & $\geq 9.0$ & 0.4 & 70 \\
Benzene & $\geq 8.0$ & 0.4 & 80 \\
Styrene & & &
\end{tabular}

\subsection{The degree of adsorption}

For inert substances with low saturated vapor pressure (such as oil and styrene), the adsorption of the tested materials is inversely proportional to the mass per unit area (area density). Each adsorbent is saturated in $20 \mathrm{~s}$, and the adsorption does not change significantly with time. Under the static conditions of the chamber, the adsorption is related to the saturated vapor pressure of HNS. Also, several adsorbents were found to exhibit the stronger adsorption for substances with larger saturated vapor pressure (See Figure 1). 

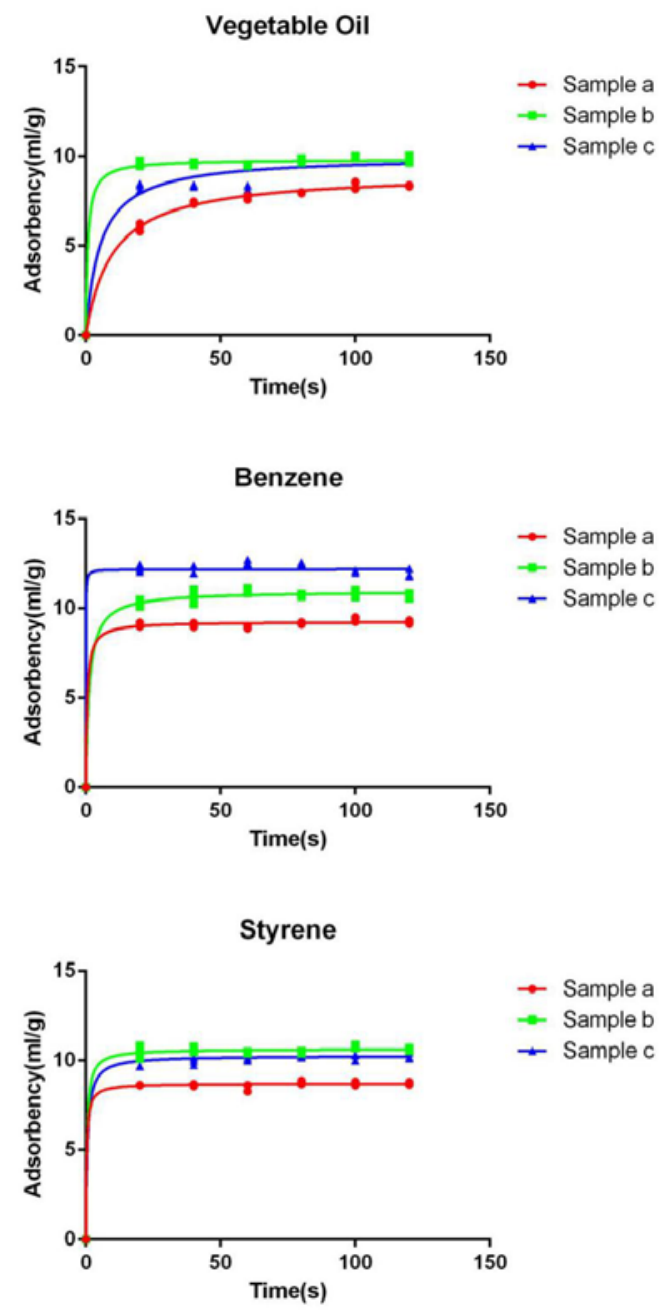

Fig. 1. The degree of Adsorption of various adsorbents

\subsection{Adsorption rate}

For inert substances with low saturated vapor pressure (oil and styrene), the adsorption rate of the adsorbents is inversely proportional to the mass per unit area (area density). The adsorption rate of several adsorbents shows a decreasing trend with the increase of adsorption time. When the adsorption is saturated, the adsorption rate of each material gradually decreases to a stable level (See Figure 2). 

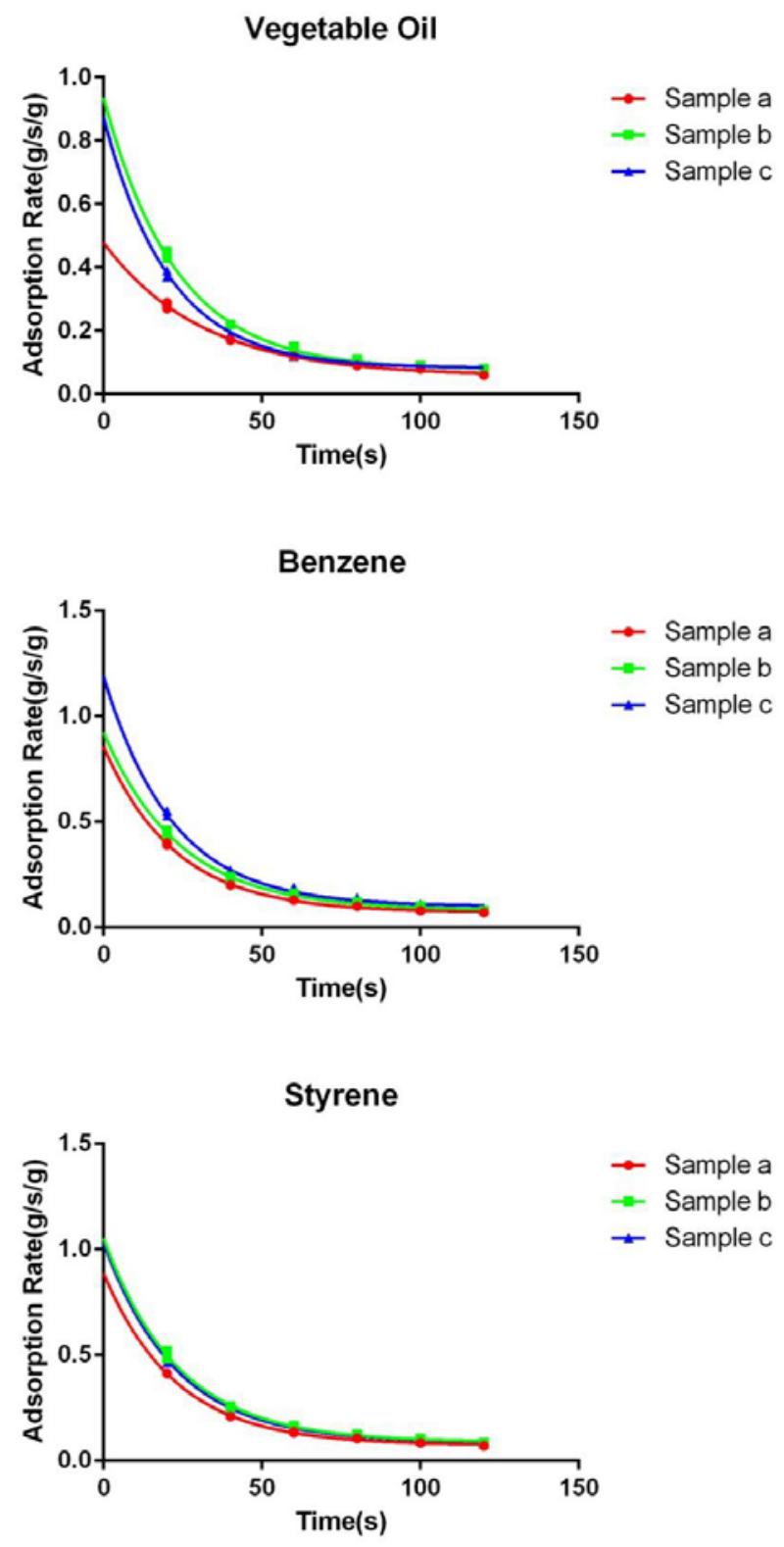

Fig. 2. The Adsorption rate of various adsorbents

\subsection{Conservation rate}

The adsorbents exhibit a good adsorption retention rate for "inert substances" with higher viscosity and lower saturated vapor pressure. The adsorption retention rate can reach more than $95 \%$. With the increase of the shaking time, the overall adsorption retention rate of benzene shows an upward trend. The saturated vapor pressure of benzene is relatively high. 
However, benzene is quite easy to evaporate compared to other substances. During the shaking process, these tested materials can adsorb benzene vapor. With the increase of shaking time, the overall adsorption retention rate of styrene decreases. Benzene exhibits certain volatility. After being adsorbed by the adsorbent, benzene cannot be completely evaporated into the air by the adsorbent (See Figure 3).
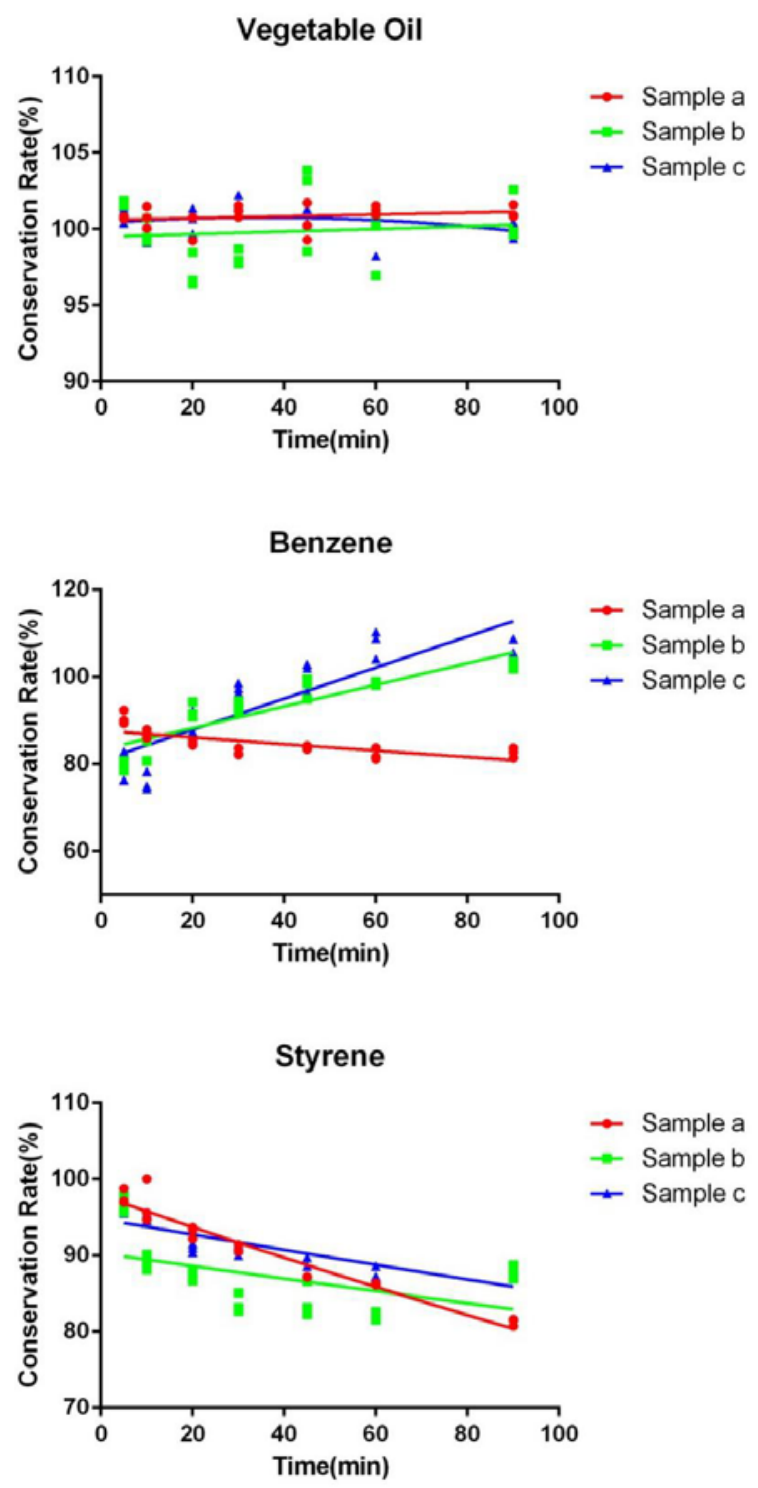

Fig. 3. The conservation rate of various adsorbents. 


\section{Acknowledgement}

Author Contributions: Methodology, H.Y. Wang, W. Chang, Y.J. Tian; writing-original draft preparation, H.Y. Wang; writing-review and editing, W. Chang, Y.J. Tian ; Experiment, H.Y. Wang, Y.J. Tian. All authors have read and agreed to the published version of the manuscript.

Conflicts of Interest: The authors declare no conflicts of interest.

\section{References}

[1] International Maritime Organization. 1990 International Convention on Oil Pollution Preparedness, Response and Cooperation. 1990.

[2] International Maritime Organization. Protocol on Preparedness, Response and Co-operation to pollution incidents by Hazardous and Noxious Substances, 2000. 2007.

[3] IOPC FUNDS. https://www.iopcfunds.org/.

[4] International Tanker Owners Pollution Federation. Response to marine chemical incidents. Technical information paper 17.

[5] International Maritime Organization. Services and Exchange-HNS Absorption and Vapour Suppression-Submitted by the International Harbour Masters' Association (IHMA). MEPC/OPRC-HNS/TG 9/5/4. 2009.

[6] American Society for Testing and Materials. Standard Test Methods for Sorbent Performance of Adsorbents. 2012

[7] International Organization for Standardization. Ships and marine technology-Marine environment protection- Guidance on design and selection of sorbents. TC 8/SC $2 \mathrm{~N}$ 460.2016 .

[8] Absorbent Material for Oils, Liquid Fuels and Water, Canadian General Standards Board, 1996.07

[9] British Standard. Materials used for the control of liquid spillages- Part 2: Determination of water repellency or buoyancy for hydrophobic (oil sorbent) materials.

[10] British Standard. Materials used for the control of liquid spillages- Part 1: Determination of sorbency.

[11] French Association for Standardization. Determination of absorbency, NFT90-360,1997.

[12] French Association for Standardization. Determination of the absorbency of all liquids, NFT90-361,1997.

[13] French Association for Standardization. Classification of absorbent products, NFT90-362, 1998.

[14] Maritime Disaster Prevention Centre. Info Bulletin-Japanese HNS Disaster Prevention Plan.2001.

[15] Ministry of Transport of the People's Republic of China. Sorbents for ship, JT/T 560-2004. 2004.

[16] Ministry of Transport of the People's Republic of China. Oil adsorption boom, JT/T 864-2013. 2013.

[17]Environmental protection center of Ministry of Transport. MDHX400SS chemical adsorbent. 2012. 
[18] Du, X.X. Study on the application performance of a new type foam for the adsorption of oil and hazardous chemicals. Dalian Maritime University. 2012.

[19] MEPC/OPRC-HNS/TG 10/5/4. 